\title{
Electroconvulsive shock and learned helplessness in rats
}

\author{
C. W. BRETT and THOMAS A. BURLING \\ Virginia Polytechnic Institute and State University, Blacksburg, Virginia 24061 \\ and
}

\author{
W. B. PAVLIK \\ University of Georgia, Athens, Georgia 30602
}

\begin{abstract}
Three experiments evaluated the effects of a single electroconvulsive shock in alleviating the learned helplessness effect in rats. The experiments differed primarily in terms of the location of the ECS treatment in the experimental sequence of events. In Experiment 1, ECS was given following helplessness training and testing and was evaluated during a retesting phase; in Experiment 2, ECS was given either immediately after helplessness training or immediately before helplessness testing; and, in Experiment 3, ECS was given prior to helplessness training. In all three experiments, significant helplessness effects occurred for subjects not receiving ECS but were absent in subjects receiving ECS. The data were compared with expectations arising from both amnesia-inducing and biochemical-change interpretations of the effects of ECS.
\end{abstract}

The term "learned helplessness" (LH), used descriptively, refers to the finding that subjects exposed to noncontingent, inescapable aversive events, such as electric shock, subsequently show performance decrements in contingent escape/avoidance tasks when compared with either naive controls or subjects who previously had experienced identical amounts of escapable shock (cf. Maier \& Seligman, 1976). Seligman (1975) has proposed that LH in animals may serve as a laboratory model of human depression, especially reactive depression; the proposal stems from a series of parallels between helpless animals and human depressives in both the behavioral symptoms shown and the presumed etiologies of such symptoms.

The present experiments were intended to shed further light on the parallels between $\mathrm{LH}$ and human depression, particularly with respect to the "therapeutic" effects of electroconvulsive shocks (ECS). It has been known for a long time that ECS is therapeutically effective with a majority of human depressives (e.g., Kolb, 1968; Nystrom, 1964; Sargent \& Slater, 1972). If the LH phenomenon is to constitute an adequate model for human depression, then one would expect ECS should also alleviate the behavioral symptoms that define the LH phenomenon in laboratory animals. This expectation is supported

Experiments 1 and 2 were taken from the doctoral dissertation of C.W.B. under the direction of W.B.P. Experiment 3 was conducted by T.A.B. and W.B.P. C. W. Brett is now at Southwestern State Hospital, Marion, Virginia. Reprint requests should be sent to W. B. Pavlik, who is at the Department of Psychology, University of Georgia, Athens, Georgia 30602. by the findings of Dorworth and Overmier (1977) in an experiment with dogs. They found that a series of six ECS treatments resulted in marked improvements in shuttlebox performance of three of six dogs that previously had appeared helpless in the same shuttlebox test situation following prior experience with inescapable shocks in a Pavlovian harness. Four similarly helpless dogs that were not given ECS treatments remained helpless during similar later tests. Dorworth and Overmier interpret their findings in terms of previous reports (e.g., Essman, 1972; Weiss, Glazer, \& Pohorecky, 1976) that brain-norepinephrine levels are affected in opposite directions by inescapable shocks (norepinephrine decreases) and ECS (norepinephrine increases). Alternative interpretations of ECS effects in such situations, focusing upon memorydisrupting effects of ECS, are also possible (see Discussion below).

The present experiments were designed to examine ECS effects in the $\mathrm{LH}$ situation and to extend the Dorworth and Overmier (1977) findings in two ways. The first was to examine such effects in the rat rather than the dog. The second was to determine the effects, if any, of manipulating the point, during the temporal sequence of training and testing, at which ECS treatments are introduced.

\section{EXPERIMENT 1}

It is necessary to distinguish among training, testing, and retesting phases of $\mathrm{LH}$ experiments. The training phase consists of some initial set of events in which helpless animals are given experiences with noncon- 
tingent, inescapable aversive events. Control (nonhelpless) animals typically receive either no trainingphase experiences or experiences with contingent, escapable aversive events. The testing phase subsequently tests for the effects of the training phase in terms of the animal's performance on some task involving contingent, escapable aversive events. The retesting phase, when used, is a subsequent repetition of the testing phase and is used to examine the reliability and/or persistence of effects. It should be noted, however, that performance during the retesting phase may be influenced by events experienced during the testing phase as well as by events experienced during the temporally more remote training phase. Dorworth and Overmier (1977) introduced ECS treatments between their testing and retesting phases and, as noted above, observed improved performance during retesting in three of the six dogs treated. Their procedure presumably duplicated what happens in the real world with human depressives. The human depressive presumably has some initial set (training phase) of failure or other experiences that result in a subsequent display (test phase) of symptoms leading to a diagnosis of depression. Therapeutic interventions such as ECS subsequently are introduced, and their effects are assessed by any still-later (retest phase) change in symptoms. In Experiment 1, rats were given a training phase in a Skinner box involving escapable shock, inescapable shock, or no-shock conditions and were subsequently given a testing phase in a shuttlebox to determine whether LH had developed. Subsequent to the testing phase, half of the animals in each training-phase condition were given a single ECS treatment. All animals subsequently were given a retesting phase in the shuttlebox.

\section{Method \\ Subjects. Forty-eight experimentally naive male hooded rats of the Long-Evans strain served as the subjects. All the subjects were from the Virginia Polytechnic Institute and State University animal colony, were between 90 and 110 days old at the time of the experiment, and were housed in individual cages with con- tinuous access to food and water.}

Apparatus. The subjects received the training phase in two fully automated $30 \times 23 \times 26 \mathrm{~cm}$ Skinner boxes with retractable levers and mounted in sound-attenuating chambers. Both boxes were illuminated continuously by two houselights and contained a speaker that provided continuously a $75-\mathrm{dB}$ white noise for masking purposes. The floor of each box consisted of .3-cm stainless steel grids, located $1.0 \mathrm{~cm}$ apart, through which could be delivered a 1.0-mA scrambled footshock from Grason-Stadler Model 700 shock generators.

Testing and retesting phases were conducted in a two-way shuttlebox, consisting of two $22 \times 10 \times 15 \mathrm{~cm}$ chambers separated by a stainless steel wall in which was centrally located an $8 \times 13 \mathrm{~cm}$ opening. The opening was covered by a manually operated plywood guillotine door. The floor of each chamber consisted of $.2-\mathrm{cm}$ stainless steel grids, located $1.0 \mathrm{~cm}$ apart, through which could be delivered a 1.0-mA scrambled footshock from the same shock sources that supplied the Skinner boxes. The shuttlebox was illuminated by two 7.5-W white light bulbs and was contained in a sound-attenuating chamber that had, in one wall, a one-way vision screen through which animals could be observed. Masking noise within the chamber was provided by $80-\mathrm{dB}$ white noise from a Grason-Stadler noise generator.

The ECS was provided by a Lafayette Instruments Company shock source, which delivered a $40-\mathrm{mA}, 1,200-\mathrm{V}$ ac shock for $5 \mathrm{sec}$. The ECS was delivered through ear clips made of alligator clips wrapped in gauze that had been soaked in an isotonic saline solution.

Procedure. On the day prior to the training phase, each animal was handled for a few minutes and then assigned randomly to one of three training conditions $(n=16)$-escapable-shock training (E), inescapable-shock training (I), or no-shock training controls (C). The subjects from Groups E and I were run together in pairs during the training phase, one subject from each group. The shock circuits for the two Skinner boxes were wired in parallel, so that identical durations of footshock were delivered to both boxes on each trial. A single training session of 80 trials was administered; intertrial intervals varied, with a mean of $60 \mathrm{sec}$. Trial duration was controlled by the subject in Group E. Initially, a single barpress was required to terminate shock in both boxes. However, after five consecutive trials with escape latencies less than $5 \mathrm{sec}$, the response requirement to terminate a trial was increased to two barpresses (FR 2); similarly, after five consecutive trials with FR 2 latencies of less than $5 \mathrm{sec}$, the response requirement was increased to an FR 3, at which it remained for the duration of the training session. This procedure was a modified version of that used by Seligman and Beagley (1975). The subject from Group I could press the lever in its Skinner box, but such barpresses had no programmed consequences other than being counted. The training phase for subjects from Group $C$ consisted of confining each subject in one of the Skinner boxes for $105 \mathrm{~min}$, which was the mean duration of the training sessions for subjects in Groups E and I. No shocks were administered to Group C subjects.

The testing phase in the shuttlebox was conducted approximately $24 \mathrm{~h}$ following the training phase. Each rat was tested individually. The rat was placed into the shuttlebox, facing away from the door, and, after a 1-min exploration period, the door was opened, initiating the first trial. Lifting the door turned on a $1,000-\mathrm{Hz}$ $80-\mathrm{dB}$ warning stimulus that was followed, $10 \mathrm{sec}$ later, by the delivery of a $1.0-\mathrm{mA}$ scrambled footshock to the grid floor of both chambers. Both the warning stimulus and the footshock continued until the rat had completed an FR 2 shuttle response-that is, the rat had to enter the opposite compartment completely and then return to the original compartment. Failure to complete the response requirement within $60 \mathrm{sec}$ resulted in the door's being lowered, the warning stimulus's and footshock's being terminated and a $60-\mathrm{sec}$ latency assigned for that trial. Each rat received 15 such trials with an intertrial interval of $30 \mathrm{sec}$.

Immediately after the completion of the testing phase, a randomly selected half of the subjects in each group $(n=8)$ was administered one ECS, while the other half received no treatment. Each rat that received ECS was taken from the shuttlebox following the 15th test trial and placed on a table top, where the ear clips were attached and ECS was delivered. Each ECS produced a full tonic-clonic convulsion followed by 3-5 min of immobility. No perseverating physical disabilities were observed in the ECS animals.

The retesting phase was conducted $24 \mathrm{~h}$ following the test phase and was identical to the test phase in all respects except that no animals received ECS following the retesting phase.

\section{Results and Discussion}

Training phase. All rats in Group E learned to barpress to escape shock, as evidenced by decreasing trial latencies and/or progressions to more stringent response requirements. The mean number of barpresses during the training session was 302.4 for Group E and 46.1 for Group I. A number of these barpresses 
occurred during the intertrial interval. The mean trial duration, which was necessarily the same for Groups $\mathrm{E}$ and $\mathrm{I}$, was $6.38 \mathrm{sec}$. Individual trial durations ranged from .1 to over $90.0 \mathrm{sec}$.

Testing phase. No avoidance response (i.e., latency less than $10 \mathrm{sec}$ ) occurred during the testing phase. Mean latencies were computed for Groups E, I, and $C$ for each of the 15 test trials and were subjected to analysis. These data are presented in the left panel of Figure 1, collapsed across blocks of three trials. It appears clear from the figure that Groups $E$ and $C$ did not differ from each other; both showed decreasing mean latencies across the 15 test trials as the rats learned to escape shock more efficiently. Group I, on the other hand, showed no improvement across the 15 test trials-clear evidence for the $\mathrm{LH}$ effect. These impressions are supported by the results of an ANOVA of the data, which yielded a significant Groups by Trials interaction effect $[F(28,588)=2.87$, $\mathrm{p}<.001]$.

Retesting phase. Mean latencies for each of the 15 retest trials were computed for each of the six subgroups and subjected to analysis; these data are presented in the right panel of Figure 1, collapsed across blocks of three trials. It appears clear from the figure that performance during the retesting phase was affected both by the ECS vs. no-ECS manipulation and by the $E, I$, and $C$ conditions of the training phase. Specifically, in the no-ECS groups, performance during the retesting phase seems to represent simply carry-over effects from the test phase; Groups $E$ and C continued to escape shock rapidly, while Group I continued to show long latencies without improvement-a continuation of the $\mathrm{LH}$ effect seen during the test phase. On the other hand, the ECS groups, regardless of performance at the end of the test phase, showed highly similar performance during the retesting phase-slow latencies at the start of retesting fol-

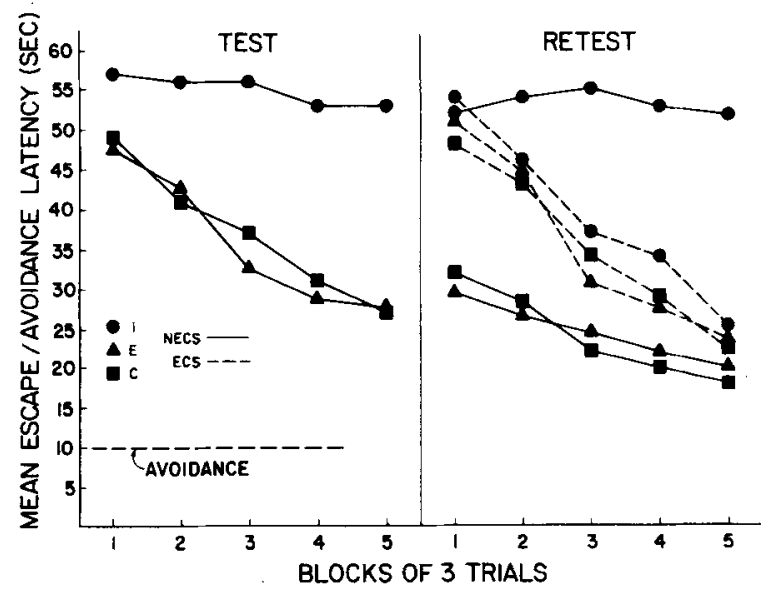

Figure 1. Mean shuttlebox latencies across blocks of three trials during the testing phase and retesting phase for Groups E, I, and C. Retesting followed either ECS treatment or no such treatment (NECS). lowed by progressively shorter latencies as the 15 retest trials occurred. Those subjects from Group I that received ECS may be said to show an attenuation of, or recovery from, LH. However, the subjects from Groups E and C that received ECS also were affected by the treatment, showing a temporary loss of efficient escape performance followed by progressive improvements back to the level attained during the testing phase.

These observations are supported by various statistical analyses. Within-subjects $t$ tests were used to compare performances for the last three-trial block of the testing phase with the first three-trial block of the retesting phase. Only Groups C-ECS and E-ECS showed significant $(p<.05)$ increases in latency from the end of testing to the start of retesting; no other groups changed significantly. An ANOVA of the data of the retesting phase yielded a significant Groups by ECS/No-ECS interaction $[F(2,42)=8.93, p<.001]$ and a significant ECS/NoECS by Trials interaction $[\mathrm{F}(14,588)=9.35, \mathrm{p}<.001]$. The source of the latter interaction is apparent in Figure 1; the ECS groups all show progressive improvements across trials, while the no-ECS groups showed no changes in latencies. The source of the former interaction also appears clear from Figure 1; no group differences exist among the three ECS groups, but Group I clearly differs from Groups E and $\mathrm{C}$ among the no-ECS groups.

Certain results of Experiment 1 clearly support the expectation from the $\mathrm{LH}$ model of depression that ECS should have therapeutic effects upon LH in animals. Those animals in Group I that were helpless during the testing phase and subsequently received a single ECS were clearly not helpless during the retesting phase when compared with helpless animals that had not received an ECS. However, an unexpected finding was that ECS produced effects in the nonhelpless subjects from Groups $\mathrm{E}$ and $\mathrm{C}$ which led to performances during retesting that were virtually identical with the performance of the ECS subjects from Group I. Furthermore, the performances of all three ECS groups during retesting were highly similar to the performance of Group $\mathrm{C}$ during the testing phase, suggesting that ECS animals during retesting were acting as if they had had no prior experiences with the testing and/or training phases of the experiment. The implications of this for interpretations of ECS effects in this situation are discussed below.

\section{EXPERIMENT 2}

ECS was administered between the testing and retesting phases of Experiment 1. Since the ECS followed both the training and testing phases of that experiment, it is unclear whether the observed ECS effects represent changes in the normal effects of the training phase alone or in the combined effects of the 
prior training and testing phases. Experiment 2 addressed this question by duplicating the training and testing phases of Experiment 1 and interpolating ECS treatments between the training and testing phases. No retesting phase was used.

\section{Method}

Subjects. The subjects were 72 naive male rats like those described in Experiment 1.

Apparatus. The apparatuses used were exactly the same as those employed in Experiment 1.

Procedure. The procedures used were identical to those described for the training and testing phases of Experiment 1, with the following exceptions: Twenty-four rats were assigned randomly to each of the three training conditions-E, $I$, and $C$. Onethird $(n=8)$ of the rats in Groups $E$ and I were given a single ECS immediately following the completion of the training phase; one-third were given a single ECS 30 min prior to the start of the testing phase (i.e., $23.5 \mathrm{~h}$ following the completion of the training phase), a condition referred to hereafter as the delayed ECS condition; and the remaining one-third of the rats in Groups $E$ and I did not receive an ECS treatment. The subjects in Group C were not preexposed to the training phase environment in this experiment; the testing phase for Group C occurred $24 \mathrm{~h}$ following a single ECS for one-third of the animals, $30 \mathrm{~min}$ following ECS for one-third, and without ECS for the remaining one-third.

\section{Results and Discussion}

Training phase. As in Experiment 1, all rats in Group $E$ learned to barpress to escape shock. The mean number of barpresses during the training session was 311.3 for Group E and 29.2 for Group I. The mean duration of shock per trial was $4.39 \mathrm{sec}$.

Testing phase. The latency data were summarized and analyzed as in Experiment 1 and are presented, collapsed across blocks of three trials, in Figure 2. The middle panel of Figure 2 presents the data of of those groups that did not receive the ECS treatments, and the data clearly suggest that a LH effect was produced; Group I shows very slow escape

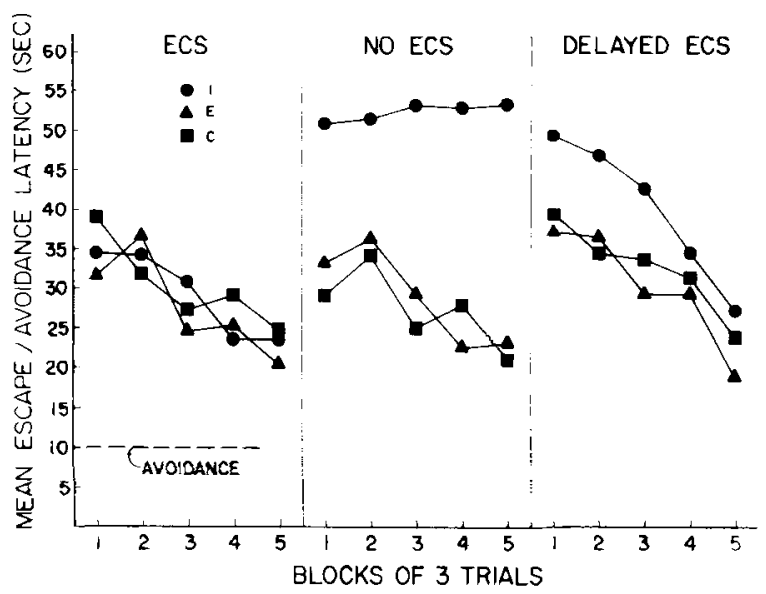

Figure 2. Mean shuttlebox latencies across blocks of three trials during the testing phase in Groups $\mathbf{E}, \mathbf{I}$, and $\mathbf{C}$ following immediateECS (left panel), delayed-ECS (right panel), or no-ESC treatments (middle panel). latencies without improvement, while Groups $E$ and C show shorter escape latencies with improvement across the 15 test trials. The data from the three groups receiving ECS immediately following training are presented in the left panel of Figure 2 . The results are highly similar to those of the ECS groups in Experiment 1 during the retesting phase. No group differences exist, and all three groups show relatively rapid and improving escape responses. Again, ECS seems to have alleviated the $\mathrm{LH}$ effect. The data from the delayed-ECS groups are presented in the right panel of Figure 2, and they present a mixed set of results. Groups $E$ and $C$ in the delayed-ECS condition were generally slower across the testing session than were the equivalent groups in the immediate ECS or no-ECS conditions. While Group I in the delayed-ECS condition gave the appearance of an $\mathrm{LH}$ effect early in the testing phase, it showed considerable improvement as the testing phase continued and was not very different from Groups $\mathrm{E}$ and $\mathrm{C}$ by the end of the 15 test trials.

The effects described above were generally confirmed by the results of an overall ANOVA, which yielded a significant Groups by ECS Treatments interaction effect $[F(4,63)=5.21, p<.01]$. In spite of the fact that all groups except Group I/no-ECS showed shorter latencies across trials, neither the Groups by Trials nor the Groups by Conditions by Trials interaction effects were significant. Simple effects analyses indicated that significant group differences existed in the no-ECS condition $[F(2,63)=$ $20.69, \mathrm{p}<.001]$ but not in the immediate- or delayedECS conditions, and that a significant ECS treatments effect existed among the I groups $[F(2,63)=13.85$, $\mathrm{p}<.01$ ] but not among the $\mathrm{E}$ and $\mathrm{C}$ groups.

The results of the no-ECS and immediate-ECS conditions generally confirm and extend the findings of Experiment 1. The large LH effect seen in the noECS groups is eliminated following the administration of a single ECS immediately after helplessness training, suggesting that the effects of ECS in such situations are upon the residues of the original training experiences and are not dependent upon any prior display of symptoms.

On the other hand, the results of the delayed-ECS condition are less clear. Groups $\mathrm{E}$ and $\mathrm{C}$ both showed (nonsignificant) longer latencies than did comparable groups in the no-ECS or immediate-ECS conditions, suggesting some proactive disabling effects upon shuttlebox performance when ECS is given only $30 \mathrm{~min}$ prior to testing. And, while it appears that delayed ECS attenuated the expected LH effect in Group I, it did so much less dramatically than did immediate ECS. Inspection of individual-subject data indicates that four of the eight subjects in Group I showed large deficits in escape responding (i.e., latencies greater than $50 \mathrm{sec}$ ) over the first seven trials of testing, after 
the scores of interest. Group NECS-I was significantly slower than Group NECS-E $(U=10, p<.01)$, an LH effect. Also, Group NECS-I was nonsignificantly slower than Group ECS-I $(U=17, p=.065)$, indicating an attenuating effect of ECS on the LH effect. Other differences of interest were not significant.

\section{GENERAL DISCUSSION}

The results of the present experiments lend some support to the LH model of human depression by showing that an effective therapeutic intervention with depression, ECS, also functions under some conditions to alleviate the $\mathrm{LH}$ effect in laboratory animals. As such, the findings support prior findings by Dorworth and Overmier (1977) and extend those findings to the laboratory rat and to different temporal locations of the ECS treatment within the training and testing sequences of typical LH experiments.

Among the diverse interpretations offered for the LH effect (see Maier \& Seligman, 1976), prominent are the norepinephrine-depletion hypothesis of Weiss (Weiss, Glazer, \& Pohorecky, 1976) and the "cognitive learned helplessness"' hypothesis of Seligman (1975). According to the former interpretation, a series of inescapable shocks produces a set of physiological changes in the animal, including a reduction in wholebrain norepinephrine levels that results in a reduction in motor cortex activity and an accompanying deficiency in initiating voluntary responses. The $\mathrm{LH}$ effect is then seen as the direct consequence of these deficiencies in response production. According to such an interpretation, LH effects should be reversed by treatments that tend to restore brain norepinephrine to normal levels.

In contrast, Seligman's cognitive interpretation of the $\mathrm{LH}$ effect assumes that animals exposed to noncontingent, inescapable aversive events learn that there is no relationship between such events and their own responses, and develop the expectation that voluntary responding will be ineffective in altering future significant events. This presumably leads both to a deficiency in response initiation and to a failure to perceive and/or learn any response-outcome contingencies that are encountered. From this perspective, the alleviation of the $\mathrm{LH}$ effect would require a set of learning experiences designed to reverse the "helplessness" expectations. This interpretation also suggests, however, that $\mathrm{LH}$ effects could be alleviated if the subjects could be induced to "forget" the experiences that gave rise to the helplessness expectations.

Interestingly enough, prior work with ECS indicates that it has effects that suggest it to be a therapeutic agent for the $\mathrm{LH}$ effect from both of the above hypotheses. On the one hand, ECS is known to produce increases in brain norepinephrine levels (e.g., Vogel \& Haubrick, 1973); on the other hand, ECS is well known as an amnestic agent (e.g., Lewis, 1969).
Thus, therapeutic effects of ECS on the helpless animals in the present experiments would be expected from either of the above viewpoints of the LH effect.

Some findings from the present set of experiments appear consistent with each of the above hypotheses, and some possibly with neither. ECS has been shown to produce amnesia for a learning experience if it occurs either shortly after the original learning experience or shortly after a "reminder" of that learning experience (cf. Lewis, 1979). A large therapeutic effect of ECS was seen in the immediate-ECS condition in Experiment 2, in which subjects received ECS immediately following helplessness training, and in Experiment 1, in which subjects received ECS immediately following a test phase that should have served as a reminder of the earlier helplessness-training phase. Both phases involved the same level of footshock, a frequently used reminder (Lewis, 1979). Thus, in both these cases, one should expect amnesia for the helpless training, possibly coupled with any persisting effects of increased brain-norepinephrine levels, to produce the observed effects.

In contrast, delayed ECS in Experiment 2 produced a less dramatic alleviation of helplessness symptoms. In that condition, ECS was given $23.5 \mathrm{~h}$ following helplessness training, and with no apparent reminders preceding the ECS. No amnesia for helplessness training should be expected in this case. However, the ECS in this case occurred only $30 \mathrm{~min}$ prior to the test phase, and one might expect that any effects of increased brain-norepinephrine levels should be apparent under such conditions.

Finally, in Experiment 3, ECS appears to have affected some subjects but not others. In this case, ECS was given $30 \mathrm{~min}$ prior to helplessness training and $24 \mathrm{~h}$ prior to testing. Since helplessness training had not yet been experienced, no retrograde amnesia for such experiences could have developed. However, it is possible that animals are sufficiently "disturbed" during a brief time following an ECS that they have difficulty learning the "helplessness expectations" that Seligman suggests underlie the LH phenomenon. It is also possible, of course, that any perseverating brain-norepinephrine changes induced by the ECS in Experiment 3 could have produced the observed results.

\section{REFERENCES}

Dorworth, T. R., \& Overmier, J. B. On "learned helplessness": The therapeutic effects of electroconvulsive shocks. Physiological Psychology, 1977, 5, 355-358.

Essman, W. B. Neurochemical changes in ECS and ECT. Seminars in Psychiatry, 1972, 4, 67-79.

Kolb, L. C. Noyes' modern clinical psychiatry (7th ed.). Philadelphia, Pa: Saunders, 1968.

LEwIS, D. J. Sources of experimental amnesia. Psychological Review, 1969, 76, 461-472.

Lewis, D. J. Psychobiology of active and inactive memory. Psychological Bulletin, 1979, 86, 1054-1083. 
Maier, S. F., \& Seligman, M. E. P. Learned helplessness: Theory and evidence. Journal of Experimental Psychology: General, 1976, 105, 3-46.

Nystrom, S. On the relation between clinical factors and efficacy of ECT in depression. Acta Psychiatrica Scandinavica, 1964, $40,5-140$.

Sargent, W., \& Slater, E. An introduction to physical methods of treatment in psychiatry. New York: Science House, 1972.

Seligman, M. E. P. Helplessness: On depression, development, and death. San Francisco: Freeman, 1975.

Seligman, M. E. P., \& Beagley, G. Learned helplessness in the rat. Journal of Comparative and Physiological Psychology, $1975,88,534-541$.
Vogel, J. R., \& Haubrick, D. R. Chronic administration of electroconvulsive shock effects on mouse-killing activity and brain monamines in rats. Physiology \& Behavior, 1973, 11, 725-728.

Weiss, J. M., Glazer, H. I., \& Pohorecky, L. A. Coping behavior and neurochemical changes. In G. Servon \& A. Kling (Eds.), Animal models in human psychobiology. New York: Plenum Press, 1976.

(Received for publication August 15, 1979; revision accepted August 5, 1980.) 